\title{
A decision tree built with parameters obtained by computed tomographic pulmonary angiography is useful for predicting adverse outcomes in non-high- risk acute pulmonary embolism patients
}

\author{
Dong Jia ${ }^{1}$, Xue-lian Li ${ }^{2}$, Qin Zhang ${ }^{3}$, Gang Hou ${ }^{3^{*}}$ (D) Xiao-ming Zhou ${ }^{4^{*}}$ and Jian Kang ${ }^{3}$
}

\begin{abstract}
Background: Acute pulmonary embolism (APE) is one of the leading causes of death in cardiovascular disease. The 30-day mortality can still be 1.7-15\% in non-high-risk APE patients. Some non-high-risk patients can progress into the high-risk group and even die, which is referred to as an adverse outcome. Promoting the diagnosis and predictive ability of adverse short-term prognosis was still a problem that needed to be solved. Computed tomography pulmonary angiography (CTPA) may be a way to promote the predictive ability. Our aim to develop predictive tools based on parameters obtained by computed tomographic pulmonary angiography (CTPA) in the form of a decision tree for use in non-high-risk acute pulmonary embolism (APE) patients.

Methods: Adverse outcome was defined within 30 days after admission to the hospital. A decision tree was built to predict adverse outcomes based on discriminating factors screened from cardiac volume and clot characteristics from recursive partitioning analysis and compared with simplified pulmonary embolism severity index (SPESI), Bova scores and risk stratification. The area under the receiver operating characteristic curve (ROC-AUC) was used to confirm the predictive ability.

Results: A total of 38 patients with and 303 patients without adverse outcomes were enrolled. Right ventricular/left ventricular (RV/LV) volume ratio, central pulmonary artery (CPA) embolism and right atria/left atria (RA/LA) volume ratio were used as splits in the decision tree to predict adverse outcomes in all patients. The ROC-AUC was 0.858. In CPA embolism patients, a recursive partitioning analysis was performed with cardiac volume and novel clot burden, but only the obstructing area $(\mathrm{OA})$ ratio was included as a discriminating factor to build a second decision tree. The ROC-AUC for the second decision tree was 0.810. The decision trees were superior to those of sPESI, Bova scores and risk stratification, and there were no significant differences between the two decision trees.
\end{abstract}

Conclusions: A decision tree built by CTPA parameters can predict adverse outcomes in non-high-risk APE patients. Keywords: Computed tomographic pulmonary angiography, Pulmonary embolism, Pulmonary artery

\footnotetext{
*Correspondence: hougangcmu@163.com; zhouxmcmu@163.com

${ }^{3}$ Department of Pulmonary and Critical Care Medicine, First Hospital of China

Medical University, No.155, Nanjing North Street, Shenyang 110001, China

${ }^{4}$ Department of Pulmonary and Critical Care Medicine, Shengjing Hospital of

China Medical University, No. 36, Sanhao Street, Shenyang 110004, China

Full list of author information is available at the end of the article
}

(c) The Author(s). 2019 Open Access This article is distributed under the terms of the Creative Commons Attribution 4.0 International License (http://creativecommons.org/licenses/by/4.0/), which permits unrestricted use, distribution, and reproduction in any medium, provided you give appropriate credit to the original author(s) and the source, provide a link to the Creative Commons license, and indicate if changes were made. The Creative Commons Public Domain Dedication waiver (http://creativecommons.org/publicdomain/zero/1.0/) applies to the data made available in this article, unless otherwise stated. 


\section{Introduction}

Acute pulmonary embolism (APE) is one of the leading causes of death in cardiovascular disease [1,2]. Differentiating high-risk APE patients from others on the basis of hypotension [1] and treating this group of patients with reperfusion therapy to save their lives are crucial due to the greater than $50 \%$ mortality rate in this group of patients [3]. However, these steps are not sufficient, as the 30-day mortality can still be $1.7-15 \%$ in non-high-risk APE patients [4]. Some non-high-risk patients can progress into the high-risk group and even die $[1,5]$, which is referred to as an adverse outcome [6]. Predicting nonhigh-risk patients with the likelihood of adverse outcomes is paramount for selecting the appropriate treatment and decreasing the mortality of APE in the short-term.

Currently, risk stratification [1], simplified pulmonary embolism severity (sPESI) and Bova scores [7] are the three main ways to predict short-term prognosis in the non-high-risk group of APE patients. However, their predictive ability is limited: sPESI is a useful tool to identify low-risk patients; and Bova scores, which were designed to predict short-term prognosis in the nonhigh-risk group of patients, still missed the diagnosis of some patients with adverse short-term outcomes [7]. Thus, promoting the diagnosis and predictive ability of adverse short-term prognosis was still a problem that needs to be addressed. Computed tomography pulmonary angiography (CTPA) may be a way to promote the predictive ability. Based on the severity of right ventricular dysfunction under pulmonary hypertension (PH), cardiac volume analysis has been helpful for predicting prognosis $[8,9]$ by CTPA. This cardiac volumetric change leads to clot blockage, which can be evaluated by clot location and clot burden analyses. Clot location correlates with the short-term prognosis [10]. However, there is still no widely accepted method to evaluate the clot burden for predicting short-term prognosis $[10,11]$.

Based on the needs of promoting the predictive ability and the usefulness of CTPA in APE, we measured cardiac volumes and evaluated novel clot burden methods, which referred to coronary computed tomographic clot burden $[12,13]$. With these parameters described above, we developed decision trees and provided visual and simplified prediction models [14] for predicting short-term prognosis in the non-high-risk group of APE patients.

\section{Materials and methods Patient selection}

This was a retrospective study from two research centers (First Hospital of China Medical University and Shengjing Hospital of China Medical University) in Shenyang, China. Between May 2014 to Dec 2018, 424 patients were collected from initially selected patients who were diagnosed with APE by CTPA, who were $\geq 18$ years of age and non-high-risk APE patients (systolic blood pressure $\geq 90 \mathrm{mmHg}$, a systolic pressure drop by $<40 \mathrm{mmHg}$, or a systolic pressure drop $\geq 40 \mathrm{mmHg}$, but for $\leq 15 \mathrm{~min}$ ) [1]. A total of 83 patients were excluded due to the following exclusion criteria: (1) 8 patients for receiving reperfusion therapy before CTPA; (2) 20 patients without CTPA data available for the reconstruction and evaluation of the clot analysis; (3) 3 patients who were pregnant; and (4) 52 patients without cardiac troponin I (cTnI), N-terminal pro-brain natriuretic peptide (NTpro BNP) or echocardiography; ultimately, 341 patients were enrolled (Fig. 1).

\section{Clinical data}

Demographic and baseline characteristics, heart rate, systolic pressure and past disease history were measured and defined from medical records upon admission to the hospital. Short-term prognosis was defined as adverse outcomes within 30 days after admission to the hospital. A positive adverse outcome $(+)$ was defined as the occurrence of at least one of the following conditions: death; cardiopulmonary resuscitation; endotracheal intubation; vasopressor requirement for systemic hypotension (more than $5 \mu \mathrm{g}$ per kilogram); or reperfusion treatment to save the patient's life $[6,9]$. These patients were grouped into the adverse outcome $(+)$ group; otherwise, patients without adverse outcomes were identified and grouped into the adverse outcome (-) group.

\section{Risk stratification}

All enrolled patients were divided into an intermediatehigh-risk group, an intermediate-low-risk group and a

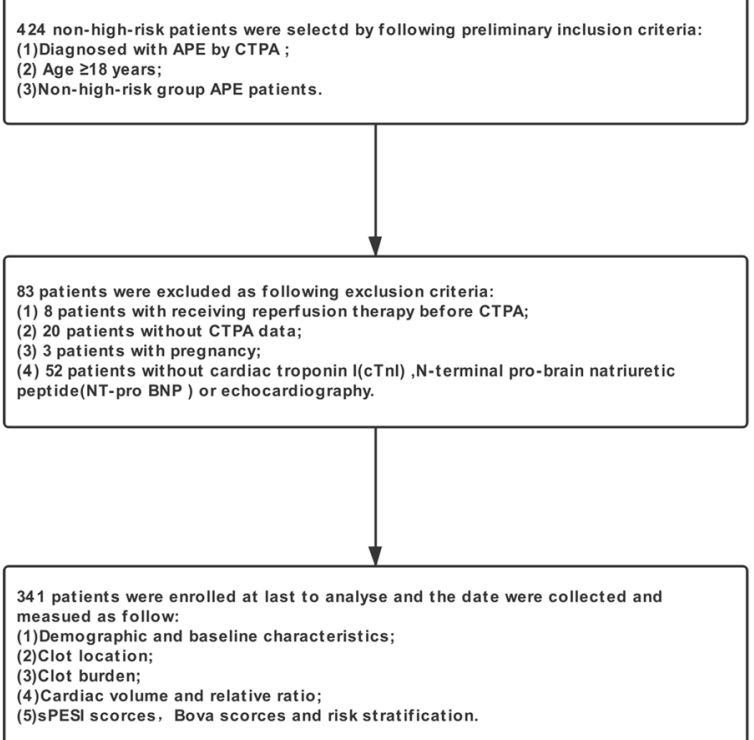

Fig. 1 Flow chart of inclusion and exclusion criteria 
low-risk group by right ventricular dysfunction (RVD), cTnI, and NT-proBNP [1]; RVD was confirmed by echocardiography [1]. cTnI $>0.04 \mu \mathrm{g} / \mathrm{L}$ (normal range 0 $0.04 \mu \mathrm{g} / \mathrm{L})$ was defined as cTnI (+); otherwise, cTnI (-) was defined [15]. NT-proBNP (+) was defined as NTproBNP $\geq 600 \mathrm{pg} / \mathrm{mL}$; otherwise, NT-pro BNP (-) was defined [1].

\section{Calculation of the prediction score}

The Bova [7] and sPESI [1] scores were calculated. The Bova score was converted into one of three classes (IIII). The sPESI score was converted into high- and lowrisk patients.

\section{CTPA acquisition}

CTPA was performed with an Aquilion KV-120 system (Toshiba Medical Systems Corporation, Tokyo, Japan) with a 64-detector row scanner. The parameters were set to $380 \mathrm{~mA}, 120 \mathrm{kV}$, and a $1-\mathrm{mm}$ section thickness for reconstruction from the thoracic inlet to the upper abdomen. An iodinated nonionic solution $(100 \mathrm{~mL})$ was injected by an automatic dual-tube high-pressure injector (Ulrich REF XD 2051; Ulrich Medical GmbH, Ulm, Germany) into the antecubital vein at $4 \mathrm{~mL} / \mathrm{s}$.

\section{Central pulmonary artery reconstruction and segmentation} All parameters were measured using Mimics Medical software (version 19.0, Mimics Medical software, Leuven, Belgium) and were recorded in the digital imaging and communications in medicine format. The central pulmonary artery (CPA) was reconstructed, including embolism from the main pulmonary artery (MPA) trunk inlet to outlets of the right and left pulmonary arteries (RPA and LPA). The centerline was established, and redundant lines were deleted manually based on the reconstructed CPA. Six center points were identified at six respective planes, including the MPA trunk inlet plane, the MPA trunk outlet plane, the LPA inlet plane, the LPA outlet plane, the RPA inlet plane and the RPA outlet plane on the reconstructed centerline. The segmenting CPA and selecting plane method refers to Schievano, S. et al. [16] and Barker, A. J.et al. [17]. The CPA was divided into four sections (MPA trunk, LPA section, RPA section and MPA triangular section) based on the

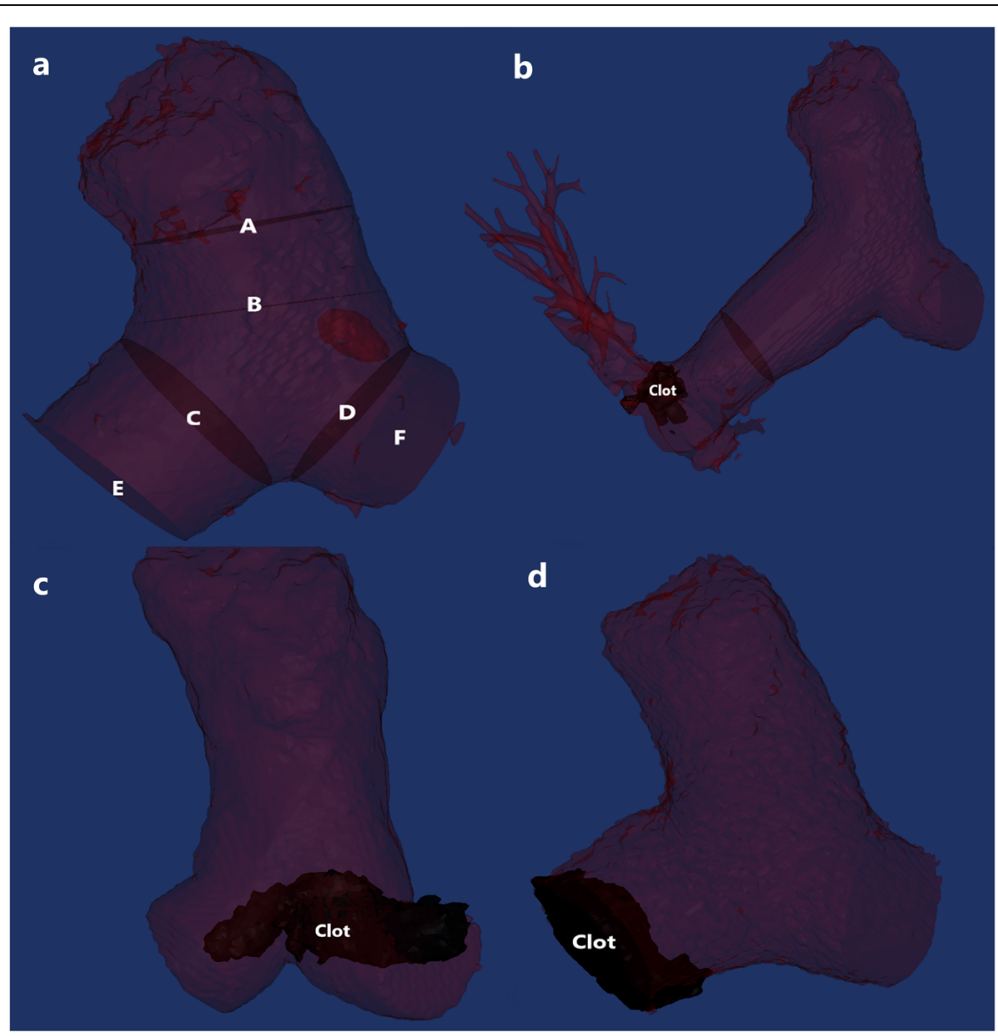

Fig. 2 Central pulmonary artery reconstruction and selected plane. a. A, main pulmonary artery inlet plane; B main pulmonary artery outlet plane; $\mathrm{C}$, right pulmonary artery inlet plane; $\mathrm{D}$, left pulmonary artery inlet plane; $\mathrm{E}$, right pulmonary artery outlet plane; $\mathrm{F}$, left pulmonary artery outlet plane; The central pulmonary artery was reconstructed from the pulmonary artery inlet plane to right and left pulmonary artery outlet planes. $\mathbf{b}$. Noncentral pulmonary artery embolism. c. Saddle-central pulmonary artery embolism. d. Central pulmonary artery embolism 


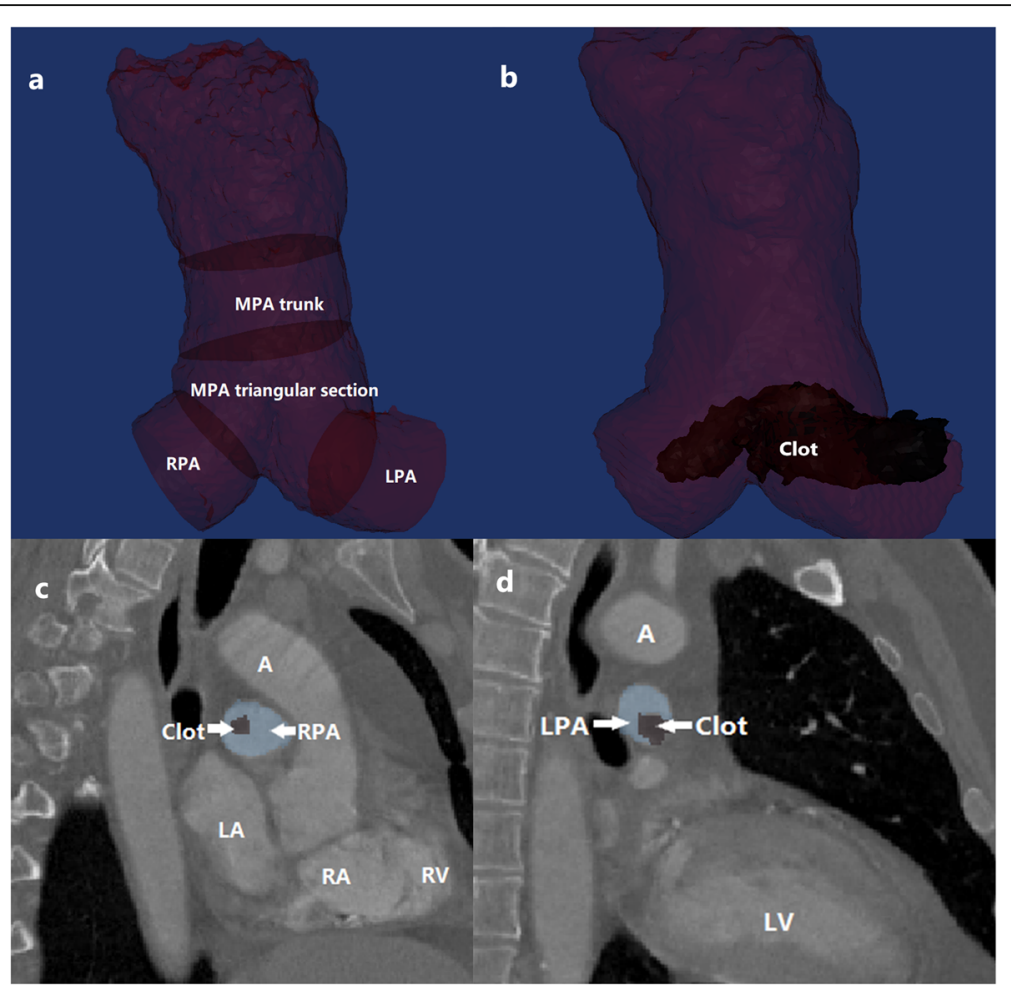

Fig. 3 Obstruction area ratio. a. The central pulmonary artery was reconstructed into four sections: main pulmonary artery trunk, MPA triangular section, and left and right pulmonary arteries. $\mathbf{b}$. The central pulmonary artery was reconstructed together with the embolismc and $\mathbf{d}$. The maximal obstruction area ratio at the right and left pulmonary was calculated as half of the total maximal obstruction area ratio at the right pulmonary artery section and the left pulmonary artery section (A: aorta; RV: right ventricle; LV, left ventricle; RA, right atrium; LA, left atrium; LPA, left pulmonary artery; RPA, right pulmonary artery). The The obstruction area ratio was calculated as half of the total maximal obstruction area ratios perpendicular to long-axis of right and left pulmonary arteries as $\mathbf{c}$ and $\mathbf{d}$

centerline, six center points and selected planes mentioned above by an orthogonal cutting function (Fig. 2.a).

\section{Clot location}

Clot locations were classified as follows: Saddle CPA embolism: embolism at the bifurcation location of the CPA [18]; CPA embolism: embolism at the CPA, including Saddle CPA embolism [19]; non-CPA embolism: embolism only at the segmental or sub segmental pulmonary artery (Fig. 2b, c, d).

\section{Clot burden evaluation}

In CPA embolism patients, clots were segmented from the reconstructed CPA. Clot burden was then evaluated

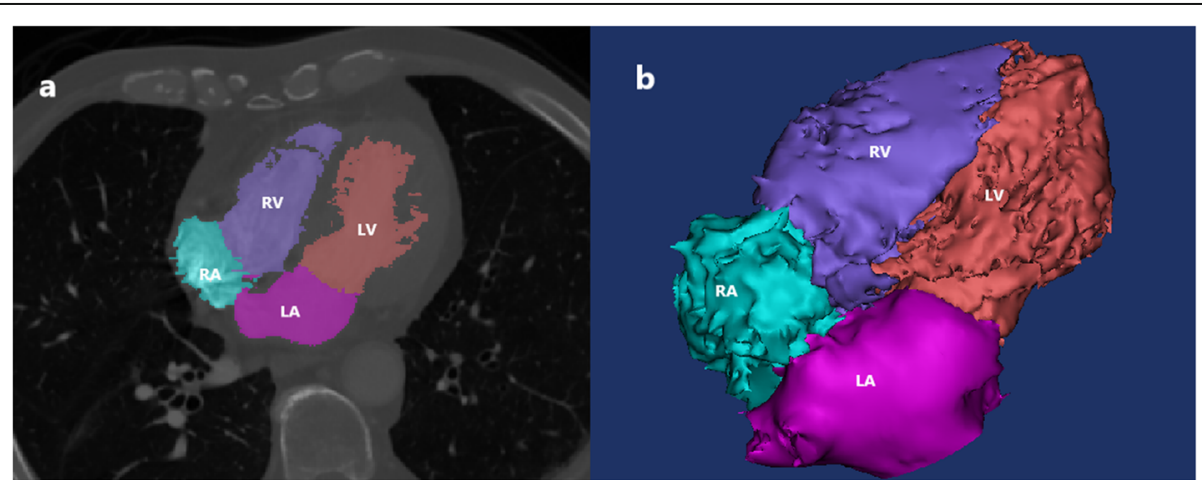

Fig. 4 Cardiac volume measurement. a. The heart chambers were differentiated as RV (right ventricle), LV (left ventricle), RA (right atrium) and LA (left atrium). b. Every heart chamber was reconstructed, the volume parameters were measured, and the relative ratios were calculated 
Table 1 Comparison of parameters between adverse outcome (+) group and adverse outcome (-) patients in all enrolled patients (mean \pm SD)

\begin{tabular}{|c|c|c|c|}
\hline & Adverse outcome $(+)$ group $(n=38)$ & Adverse outcome $(-)$ group $(n=303)$ & $p$ value \\
\hline Age (year) & $61.34 \pm 12.72$ & $60.60 \pm 14.90$ & 0.770 \\
\hline Sex (male/female) & $18 / 20$ & $136 / 167$ & 0.936 \\
\hline RA volume (ml) & $57.68 \pm 14.97$ & $54.69 \pm 15.45$ & 0.251 \\
\hline LA volume (ml) & $44.46 \pm 14.16$ & $59.69 \pm 13.85$ & $<0.001$ \\
\hline RV volume (ml) & $64.86 \pm 21.58$ & $51.05 \pm 16.57$ & $<0.001$ \\
\hline LV volume (ml) & $51.71 \pm 12.25$ & $63.57 \pm 9.93$ & $<0.001$ \\
\hline RA/LA volume ratio & $1.50 \pm 0.82$ & $0.97 \pm 0.41$ & $<0.001$ \\
\hline RV/LV volume ratio & $1.34 \pm 0.66$ & $0.82 \pm 0.30$ & $<0.001$ \\
\hline CPA embolism/non/CPA embolism & $31 / 7$ & $103 / 200$ & $<0.001$ \\
\hline Saddle CPA embolism/non-saddle CPA embolism & $9 / 29$ & $10 / 293$ & $<0.001$ \\
\hline
\end{tabular}

$R A$ right atria; $L A$ left atria; $R V$ right ventricular; $L V$ left ventricular; $C P A$ central pulmonary artery

by five methods: (1) the widest clot diameters orthogonal to the long axis of the RPA and LPA were measured, and the total of the widest diameters in the RPA and LPA was recorded as the clot maximal diameter [10]; (2) the widest clot areas orthogonal to the long axis of the RPA and LPA were measured, and the total of the widest areas in the RPA and LPA was recorded as the clot maximal area; (3) the clot lesion length was measured on the centerline relative to the lesion CPA [13]; (4) the clot aggressive volume ratio was calculated as the ratio of the clot volume relative to the volume of the pulmonary artery in CPA embolism patients [12, 13], (5) The maximal ratio of embolism area to the artery area of corresponding plane, was calculated at RPA and LPA perpendicular to long axis respectively, which represented the percentage of maximal obstruction of pulmonary artery. The obstruction area (OA) ratio was calculated as mean of the two maximal ratios at the RPA and LPA above mentioned in CPA embolism patients [12] (Fig. 3a, b, c, d).

\section{Measurement of cardiac volumes}

The cardiac chambers were segmented and reconstructed semi-automatically. The interatrial septum, interventricular septum and heart chamber walls were excluded, and the cardiac valves were used to segment different heart chambers and vessels. The cardiac capacity was measured according to the previous methods $[8,9]$. The left atrial (LA) volume, right atrial (RA) volume, left ventricular (LV) volume and right ventricular (RV) volume were measured. The RA/LA and RV/LV volume ratios were calculated as the RA volume relative to the LA volume and the RV volume relative to the LV volume (Fig. 4a, b).

\section{Statistical analysis}

Quantitative variables are expressed as the mean \pm standard deviation (SD) and were analyzed by Student's t-test to compare differences. Categorical variables are expressed as $(+) /(-)$ and were analyzed by $\chi^{2}$ test to compare differences. A tree-based methodology was built to model predictive prognosis factors and to identify effect modifications with recursive partitioning analysis between different variables to determine which variables were identified less easily by other regression models [20]. Recursive partitioning analysis was used to evaluate all possible dichotomous splits for all potential decisional factors and choose the splits providing the optimal separations by binomial data [8,21]. After each separation, the process was applied to each subgroup recursively until the subgroups reached a minimum size or no improvement could be made [22]. After pruning, a simple model was selected for clinical practice. Area under the receiver operating characteristics curve (ROCAUC) analysis was used to evaluate the predictive ability of the decision tree predictive model. Predictive abilities

Table 2 Comparison of parameters between different risk stratification groups (mean \pm SD)

\begin{tabular}{llll}
\hline Parameter & Intermediate-high-risk group $(n=69)$ & Intermediate-low-risk group $(n=77)$ & Low-risk group $(n=195)$ \\
\hline Age (year) & $62.23 \pm 12.20$ & $62.42 \pm 14.55$ & $59.46 \pm 15.43$ \\
Sex (male/female) & $33 / 36$ & $35 / 42$ & $87 / 106$ \\
Adverse outcome $(+) /(-)$ & $20 / 49$ & $10 / 67$ & $8 / 187$ \\
\hline
\end{tabular}


Table 3 Comparison of parameters between different stages of Bova scores (mean \pm SD)

\begin{tabular}{llll}
\hline Parameter & Stage III $(n=27)$ & Stage II $(n=51)$ & Stage I $(n=263)$ \\
\hline Age (year) & $62.19 \pm 11.38$ & $62.45 \pm 12.93$ & $60.19 \pm 15.26$ \\
Sex (male/female) & $15 / 12$ & $24 / 27$ & $116 / 147$ \\
Adverse outcome $(+) /(-)$ & $12 / 15$ & $12 / 39$ & $14 / 249$ \\
\hline
\end{tabular}

were compared with the difference in ROC-AUC [23, 24]. A two-tailed $p$ value less than 0.05 was considered to indicate a significant difference. The statistical analysis was performed with $\mathrm{R}$ software version 3.3.2 (http://www.R-project.org) and Medical statistical software (version 15.8, Belgium).

\section{Results}

Demographics, baseline characteristics and comparisons of the measured parameters in all the enrolled patients Ultimately, 341 patients were enrolled. A total of 38 patients were defined as adverse outcome $(+)$, and 303 patients were defined as adverse outcome (-). The RV volume, RA/LA volume ratio, RV/LV volume ratio, and the ratio of CPA embolism (+) to saddle CPA embolism $(+)$ in the adverse outcome $(+)$ group were higher than those in the adverse outcome (-) group, with significance $(p<0.001)$. LA and LV volumes in the adverse outcome $(+)$ group were lower than those in the adverse outcome $(-)$ group, with significance $(p<0.001)$ (Table 1).

Risk stratification, Bova scores and different classes of s PESI scores

Risk stratification: The ratio of adverse outcomes in the intermediate-high-risk group was $40.8 \%$; the ratio of adverse outcomes in the intermediate-low-risk group was $13.0 \%$, and the ratio of adverse outcomes in the low-risk group was $4.1 \%$ (Table 2).

Bova scores: The ratio of adverse outcomes in stage III was $44.4 \%$; the ratio of adverse outcomes in stage II was $23.5 \%$, and the ratio of adverse outcomes in stage I was 5.3\% (Table 3).

sPESI score: The ratio of adverse outcomes in the high-risk group was $13.9 \%$; the ratio of adverse outcomes in the low-risk group was $2.6 \%$ (Table 4 ).

Demographics, baseline characteristics and comparisons of the measured parameters in CPA embolism patients The RV volume, RA/LA volume ratio, RV/LV volume ratio, OA ratio, clot aggressive volume ratio, clot aggressive volume ratio, clot maximal diameter and clot maximal area in the adverse outcome $(+)$ group were higher than those in the adverse outcome (-) group, with significance $(p<0.001,<0.001,<0.001,<0.001,0.001,<0.001,0.036$ and $<0.001$, respectively). The LA and LV volumes in the adverse outcome $(+)$ group were lower than those in the adverse outcome $(-)$ group, with significance $(p<0.001$ and $<0.001$, respectively) (Table 5).

\section{Decision tree and predictive ability for adverse outcome}

We developed a recursive partitioning model with cardiac volume and clot location together with sex and age. The decision tree was built based on recursive partitioning analysis. RV/LV volume ratio, CPA embolism and RA/LA volume ratio were included as discriminating factors in the decision tree for predicting adverse outcomes and formed four groups of relevance after three splits: (a) Split: all 341 enrolled patients were divided based on RV/LV volume ratio $<1.11 \quad(n=287) \quad$ or $\geq 1.11 \quad(n=54)$, with adverse outcome ratios of 3.1 and $53.7 \%$, respectively; (b) Split: patients were divided based on RV/LV volume ratio $\geq 1.11$ patients with CPA embolism $(+) /(-)(n=42 / 12)$, with adverse outcome ratios of 61.9 and $25 \%$, respectively; (c) Split: patients were divided based on RV/LV volume ratio $\geq 1.11$ patients and CPA embolism $(+)$ patients with RA/LA volume ratios $<1.28(n=23)$ or $\geq 1.28 \quad(n=19)$, with adverse outcome ratios of 39.1 and $89.5 \%$, respectively (Fig. $5 \mathrm{a}$ ). ROC-AUC built by the recursive partitioning analysis predictive model revealed that the area was 0.858 (95\%CI: 0.775-0.941) (Fig. 6).

In CPA embolism patients, we also developed a recursive partitioning analysis with cardiac volume and novel clot burden methods together with sex and age. Only the OA ratio was included as a discriminating factor for predicting adverse outcomes. The recursive partitioning analysis formed two groups of relevance: Split: 134 CPA embolism patients were divided based on an OA ratio $<0.31(n=107)$ or $\geq 0.31 \quad(n=27)$, with adverse outcome ratios of 9.3 and $77.6 \%$, (Fig. 5b). ROC-AUC revealed that the area was 0.810 (95\%CI: 0.706-0.913).

Table 4 Comparison of parameters between different sPESI scores (mean \pm SD)

\begin{tabular}{lll}
\hline Parameter & High-risk $(n=303)$ & Low-risk $(\mathrm{n}=38)$ \\
\hline Age (year) & $62.25 \pm 14.49$ & $56.21 \pm 15.40$ \\
Sex (male/female) & $133 / 170$ & $22 / 16$ \\
Adverse outcome $(+) /(-)$ & $37 / 266$ & $1 / 37$ \\
\hline
\end{tabular}


Table 5 Comparison of parameters between adverse outcome (+) group and adverse outcome (-) patients in CPE patients (mean \pm SD)

\begin{tabular}{llll}
\hline & Adverse outcome $(+)$ group $(n=31)$ & Adverse outcome $(-)$ group $(n=103)$ & $p$ value \\
\hline Age (year) & $62.42 \pm 11.16$ & $59.84 \pm 13.36$ & 0.330 \\
Sex (male/female) & $14 / 17$ & $47 / 56$ & 0.963 \\
RA volume (ml) & $58.72 \pm 15.15$ & $54.24 \pm 12.96$ & 0.143 \\
LA volume (ml) & $43.18 \pm 13.17$ & $57.45 \pm 17.65$ & $<0.001$ \\
RV volume (ml) & $67.85 \pm 22.16$ & $52.67 \pm 12.55$ & $<0.001$ \\
LV volume (ml) & $50.80 \pm 8.96$ & $62.89 \pm 9.72$ & $<0.001$ \\
RA/LA volume ratio & $1.56 \pm 0.82$ & $1.01 \pm 0.34$ & $<0.001$ \\
RV/LV volume ratio & $1.39 \pm 0.67$ & $0.85 \pm 0.25$ & $<0.001$ \\
Obstruction area ratio & $0.38 \pm 0.23$ & $0.11 \pm 12$ & $<0.001$ \\
Clot aggressive volume ratio & $0.33 \pm 0.25$ & $0.16 \pm 0.15$ & 0.001 \\
Clot lesion length (mm) & $41.09 \pm 35.32$ & $13.75 \pm 22.45$ & $<0.001$ \\
Clot maximal diameter (mm) & $35.24 \pm 24.35$ & $16.01 \pm 15.37$ & 0.036 \\
Clot maximal area (mm $\left.{ }^{2}\right)$ & $374.36 \pm 383.67$ & $78.49 \pm 139.83$ & $<0.001$ \\
\hline
\end{tabular}

$R A$ right atria; $L A$ left atria; $R V$ right ventricular; $L V$ left ventricular

The difference in the predictive ability of the two decision trees

We compared the predictive abilities in ROC-AUC of the decision trees based on the volume index and on the $\mathrm{OA}$ ratio in CPA embolism. The difference was not significant $(p=0.48)$. We also compared the predictive ability between the decision tree and the risk stratification, Bova scores and sPESI scores (Fig. 6).

\section{Discussion}

Predicting adverse outcomes in non-high-risk APE patients has been a significantly challenging problem [10]. Cardiac volume and relative ratio have also shown correlations with short-term prognosis in APE [8]. Clot blockage lead to this change of cardiac volume in substance. However, the method of evaluating severity of clot obstruction, called the clot burden, did not show a correlation with short-term prognosis in APE $[1,11]$. Instead, clot location is a widely used applicable predictor for APE prognosis [10, 19]. Our result revealed that the RV/ LV volume ratio was the first discriminating factor for predicting adverse outcomes with optimal differentiation degrees in the first decision tree of all APE patients. The RA/LA volume ratio was also a discriminating factor. The difference between the RV/LV and RA/LA volume ratios in terms of importance in the decision tree was caused by their different structures and reactions to pressure $[8,25]$. Considering that the ratio between the right and left heart volumes has a relevance as a prognostic index, it would be interesting to know which is the main contribute to the increase of the ratio: right heart volumes enlargement or left heart volumes reduction. Our results showed that the left heart volumes reduction may be the main contribute to the adverse outcomes, as there was no difference in the RA volume between adverse outcome (+) group and adverse outcome $(-)$ group whereas the LA volume of adverse outcome (+) group was smaller than that of adverse outcome (-) group. For the ventricular volume contributes, we deduced that LV contributed more than RV to the finial consequence of adverse outcome, because the hemodynamic collapses related to low cardiac output, was main manifestation of adverse outcome in APE patient. But, differentiating the weighing of the contribution of $\mathrm{LV}$ and $\mathrm{RV}$ to the $\mathrm{RV} / \mathrm{LV}$ volume ratio was difficult, because the change of the RV and LV were dependent on each other and they were interactive due to the mutual compression. The decreased LV volume was led by the increasing RV stain, but when evaluated, RV and LV changed simultaneously. LV volume might be one of the discriminatory factors of the adverse outcome, but RV/LV volume ratio was the most optimal discriminatory factor when compared to single ventricular volume in the decision tree in our study. In addition, CPA embolism was another discriminating factor in the decision tree, which revealed the correlation between the cardiac volume and clot blockage. The decision tree integrated a module that could help the clinicians screen for the potential adverse outcome $(+)$ patients.

CPA embolism correlates with prognosis [10], and it is an important discriminating factor for predicting adverse outcomes in the decision trees of our study. We evaluated the obstruction severity by half of the total maximal obstruction area ratios at the RPA and LPA sections in CPA embolism patients. In the subgroup of CPA embolism, we developed several novel methods for evaluating 


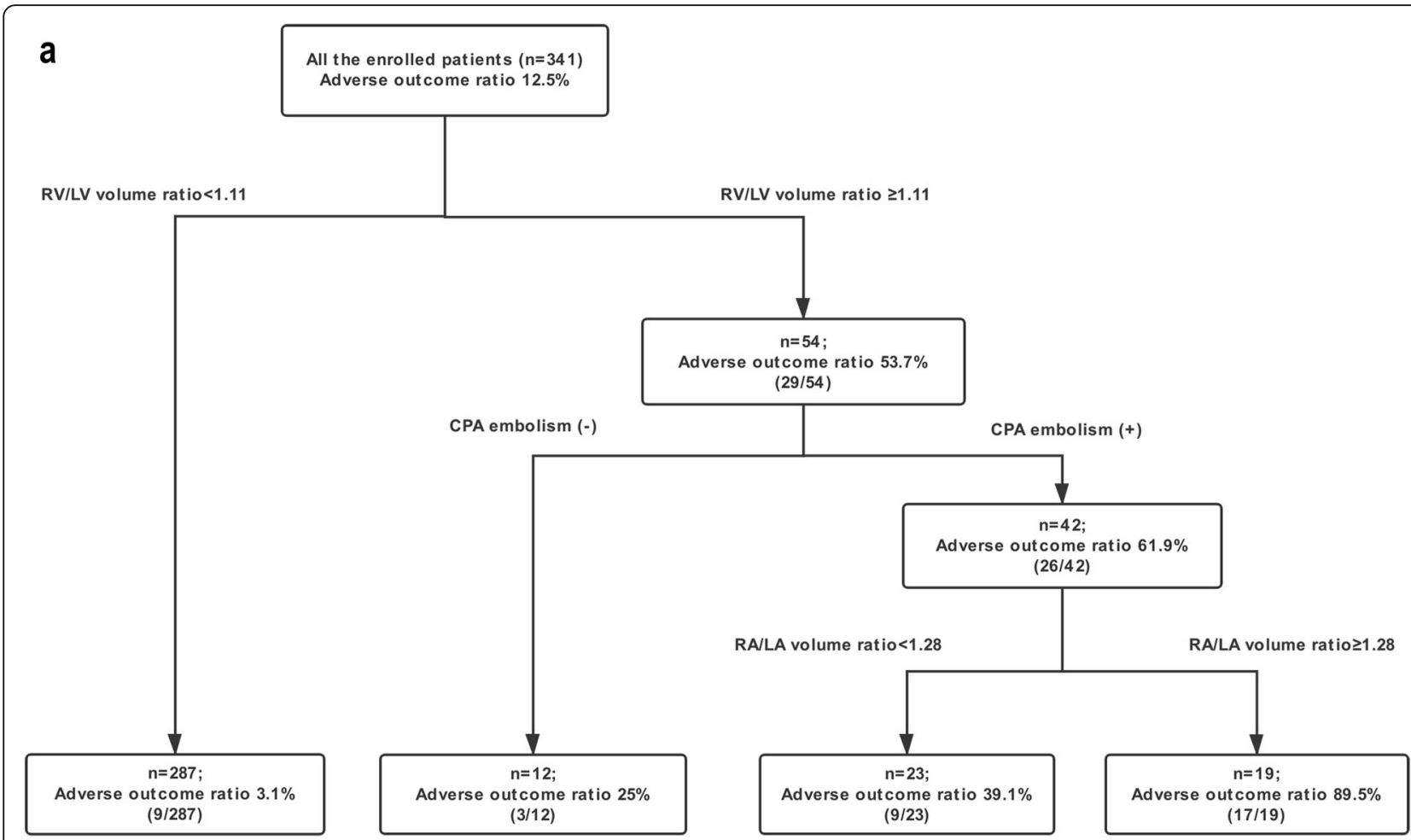

b

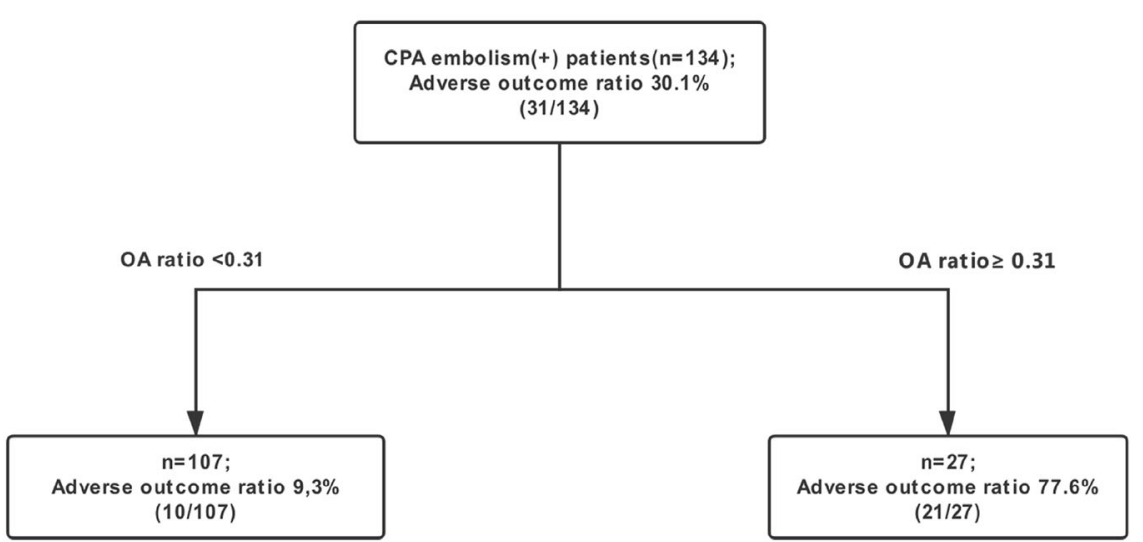

Fig. 5 Decision trees for all patients and acute pulmonary embolism patients. $\mathbf{a}$. The decision tree was developed by the RV/LV ratio, CPA embolism, and RA/LA volume ratio in the enrolled patients. b. OA ratio was optimal discriminating factor in CPA embolism patients

clot burden, including the maximal clot diameter, area, clot length, volume ratio and OA ratio, together with cardiac volume. The results showed that only the OA ratio was the optimal discriminating factor among these methods of clot burden in CPA embolism patients. The OA ratio was another way to identify adverse outcomes without measuring cardiac volume, which is a complicated process, and it could obtain a similar predictability as the decision tree built with the cardiac volume index and the CPA embolism.

Saddle CPA demonstrates embolism in bifurcation of the CPA and the clot blockage on the two sides of CPA.
However, in our analysis, saddle CPA embolism was not a discriminating factor. The reason may be explained by the larger section at CPA bifurcation section than other sections [16], while the larger section represented a reduced influence on blood flow. The frequency of saddle CPA embolism was $5.6 \%$ in our study. However, our study may underestimate the occurrence probability of saddle CPA embolism and the associated mortality, because some patients with saddle CPA embolism with a poor prognosis and severe adverse outcomes would not survive before CTPA is performed, as indicated in a previous study [26]. 


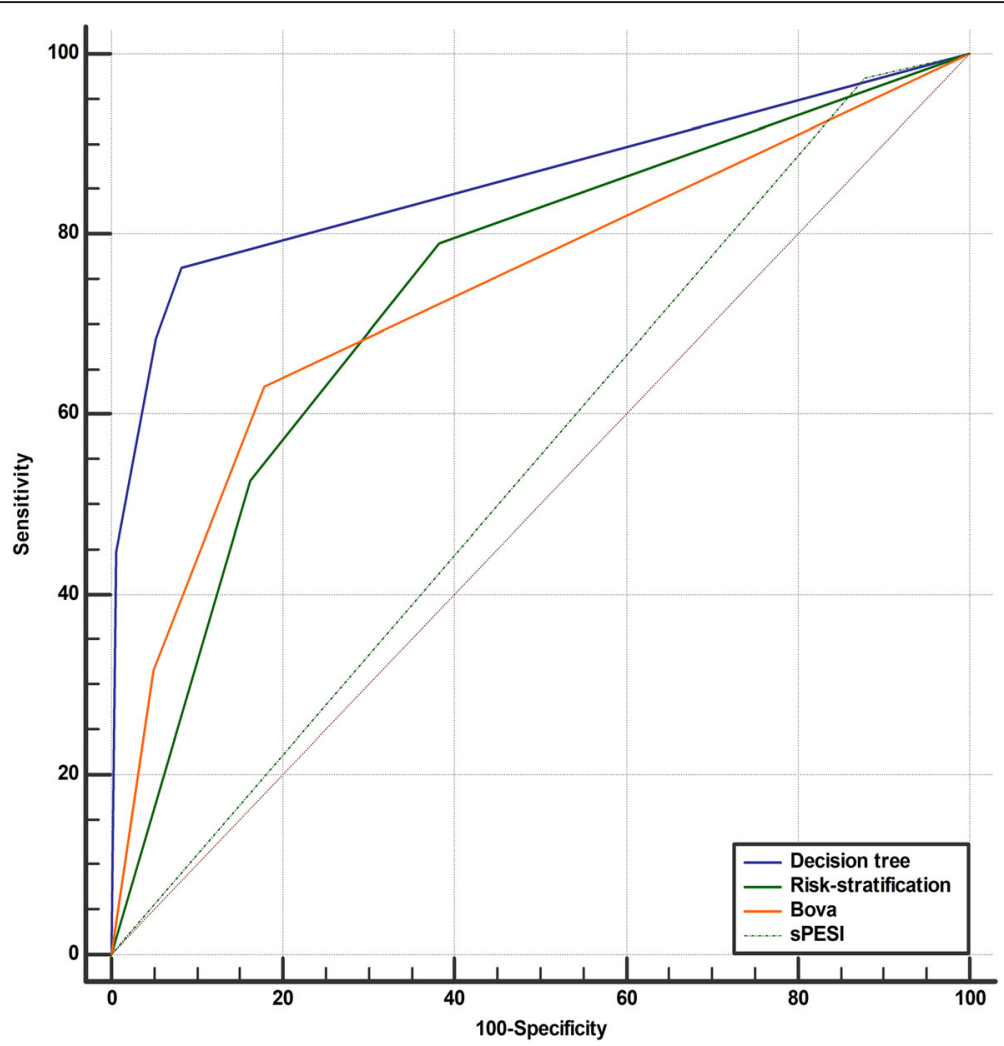

Fig. 6 The predictive abilities of the decision tree and other predictive scores. The ROC curve built by the recursive partitioning analysis predictive model for all enrolled patients revealed that the area under the ROC was 0.858 (95\%Cl: 0.775-0.941). For all enrolled patients, the ROCAUC revealed that the area of risk stratification, Bova scores and SPESI scores were 0.740 (95\%Cl: 0.690-0.786), 0.739 (95\%Cl: 0.689-0.785) and 0.548 (95\%Cl: $0.493-0.602)$, respectively. The predictive ability of the decision tree was better than others in the ROC-AUC $(0.118,95 \% \mathrm{Cl}: 0.0139-$ $0.222, p<0.05 ; 0.119,95 \% \mathrm{Cl}: 0.00306-0.234, p<0.05$ and $0.310,95 \% \mathrm{Cl}: 0.229-0.391, p<0.05$ )

Until now, traditional or classic methods of predicting the prognosis of APE have needed to be combined with clinical vital signs, such as echocardiography and biochemical indicators. As a diagnostic tool, CTPA only provides a static image, but it is still helpful for predicting adverse outcomes because relying solely on this static image is timesaving, as there is no need to wait for other results. In our study, the abilities for predicting the high probability of adverse outcome $(+)$ patients and screening for adverse outcome (-) patients were superior than risk stratification and Bova scores. Discriminating using the decision trees built in our study would be easier, faster and more accurate than traditional methods. If only the cardiac volume index is available, or the CPA embolism is negative, the decision tree built by the integration of RV/LV volume ratio, CPA embolism and RA/LA volume ratio would be helpful in the discrimination. However, when the $\mathrm{OA}$ is available as the clot characteristics, while the patient is CPA embolism $(+)$, the decision tree built with CPA embolism and OA would be easier than the cardiac volume index. Having PE diagnosis and prediction of PE severity in a single test is a potential advantage of using the decision trees built in our study.

The retrospective research design of our study limits the strength of our results. Additionally, our sample size was only 341. Different cardiac cycles could influence measurement cardiac volume. However, most studies describe measuring cardiac volume parameters with non-electrocardiographically gated computed tomographic angiography, including ours. The two decision trees in our study and the OA ratio, as a novel method for evaluating clot burden, still require further validation.

\section{Conclusion}

Following the decision tree built using the RV/LV volume ratio, the RA/LA volume ratio and identifying a CPA embolism would facilitate the prediction of adverse outcomes in non-high-risk APE patients. In the CPA subgroup of patients, measuring the OA ratio is another way to predict adverse outcomes as a supplemental method. 


\section{Abbreviations}

APE: Acute pulmonary embolism; CPA: Central pulmonary artery; cTnl: Cardiac troponin I; CTPA: Computed tomographic pulmonary angiography; LA: Left atrial; LPA: Left pulmonary artery; LV: Left ventricular; MPA: Main pulmonary artery; NT-pro BNP: N- terminal pro- brain natriuretic peptide; OA: Obstruction area; $\mathrm{PH}$ : Pulmonary hypertension; RA: Right atrial; ROC-AUC: The area under the receiver operating characteristic curve; RPA: Right pulmonary artery; RV: Right ventricular; RVD: Right ventricular dysfunction; sPESI: Simplified pulmonary embolism severity index

\section{Acknowledgments}

This article accepted language-editing service from American Journal Experts.

\section{Authors' contributions}

DJ and QZ acquired and analyzed the patient data. XL, GH and JK analyzed and interpreted the patient data. DJ, GH and XZ were major contributors in writing the manuscript. All authors read and approved the final manuscript.

\section{Funding}

The authors state that this work has not received any funding.

\section{Availability of data and materials}

The datasets used and/or analyzed during the current study are available from the corresponding author on reasonable request.

\section{Ethics approval and consent to participate}

The use of patient records was approved by the Institutional Ethical Review Board of the First Hospital of China Medical University. Patient confidentiality was maintained.

\section{Consent for publication}

Not applicable.

\section{Competing interests}

The authors declare that they have no competing interests.

\section{Author details}

${ }^{1}$ Department of Emergency, Shengjing Hospital of China Medical University, No. 36, Sanhao Street, Shenyang, China. ${ }^{2}$ Department of Epidemiology, School of Public Health, China Medical University, No.77, Puhe Road, Shenyang, China. ${ }^{3}$ Department of Pulmonary and Critical Care Medicine, First Hospital of China Medical University, No.155, Nanjing North Street, Shenyang 110001, China. ${ }^{4}$ Department of Pulmonary and Critical Care Medicine, Shengjing Hospital of China Medical University, No. 36, Sanhao Street, Shenyang 110004, China.

Received: 13 May 2019 Accepted: 12 August 2019

Published online: 19 August 2019

\section{References}

1. Konstantinides SV, Torbicki A, Agnelli G, Danchin N, Fitzmaurice D, Galie N, Gibbs JS, Huisman MV, Humbert M, Kucher N, et al. 2014 ESC guidelines on the diagnosis and management of acute pulmonary embolism. Eur Heart J. 2014;35:3033-69 3069a-3069k.

2. Zhang Z, Lei J, Shao X, Dong F, Wang J, Wang D, Wu S, Xie W, Wan J, Chen $H$, et al. Trends in hospitalization and in-hospital mortality from VTE, 2007 to 2016, in China. Chest. 2019;155:342-53.

3. Cok G, Tasbakan MS, Ceylan N, Bayraktaroglu S, Duman S. Can we use CT pulmonary angiography as an alternative to echocardiography in determining right ventricular dysfunction and its severity in patients with acute pulmonary thromboembolism? Jpn J Radiol. 2013;31:172-8.

4. Yazici S, Kiris T, Ceylan US, Akyuz S, Uzun AO, Haci R, Terzi S, Dogan A, Emre $A$, Yesilcimen $K$. The accuracy of combined use of troponin and red cell distribution width in predicting mortality of patients with acute pulmonary embolism. Wien Klin Wochenschr. 2016;128:596-603.

5. Sanchez O, Trinquart L, Colombet I, Durieux P, Huisman MV, Chatellier G, Meyer $G$. Prognostic value of right ventricular dysfunction in patients with haemodynamically stable pulmonary embolism: a systematic review. Eur Heart J. 2008;29:1569-77.

6. Konstantinides S, Geibel A, Heusel G, Heinrich F, Kasper W, Management S, Prognosis of pulmonary Embolism-3 trial I. Heparin plus alteplase compared with heparin alone in patients with submassive pulmonary embolism. N Engl J Med. 2002:347:1143-50

7. Fernandez C, Bova C, Sanchez O, Prandoni P, Lankeit M, Konstantinides S, Vanni S, Fernandez-Golfin C, Yusen RD, Jimenez D. Validation of a model for identification of patients at intermediate to high risk for complications associated with acute symptomatic pulmonary embolism. Chest. 2015;148: 211-8.

8. Galit Aviram ES, Bendet A, Shmueli H, Ziv-Baran T, Amitai Y, Friedensohn L, Berliner S, Meilik A, Topilsky Y. Prediction of mortality in pulmonary embolism based on left atrial volume measured on CT pulmonary angiography. Chest. 2016;149:667-75.

9. Kang DK, Thilo C, Schoepf UJ, Barraza JM Jr, Nance JW Jr, Bastarrika G, Abro JA, Ravenel JG, Costello P, Goldhaber SZ. CT signs of right ventricular dysfunction: prognostic role in acute pulmonary embolism. JACC Cardiovasc Imaging. 2011:4:841-9.

10. Hariharan P, Dudzinski DM, Rosovsky R, Haddad F, MacMahon P, Parry B, Chang Y, Kabrhel $C$. Relation among clot burden, right-sided heart strain, and adverse events after acute pulmonary embolism. Am J Cardiol. 2016; 118:1568-73.

11. Furlan A, Aghayev A, Chang CC, Patil A, Jeon KN, Park B, Fetzer DT, Saul M, Roberts MS, Bae KT. Short-term mortality in acute pulmonary embolism: clot burden and signs of right heart dysfunction at CT pulmonary angiography. Radiology. 2012;265:283-93.

12. Ko BS, Wong DT, Cameron JD, Leong DP, Soh S, Nerlekar N, Meredith IT, Seneviratne SK. The ASLA score: a CT angiographic index to predict functionally significant coronary Stenoses in lesions with intermediate severity-diagnostic accuracy. Radiology. 2015;276:91-101.

13. Nakazato R, Shalev A, Doh JH, Koo BK, Gransar H, Gomez MJ, Leipsic J, Park $H B$, Berman DS, Min JK. Aggregate plaque volume by coronary computed tomography angiography is superior and incremental to luminal narrowing for diagnosis of ischemic lesions of intermediate stenosis severity. J Am Coll Cardiol. 2013;62:460-7.

14. Audureau E, Chivet A, Ursu R, Corns R, Metellus P, Noel G, Zouaoui S, Guyotat J, Le Reste PJ, Faillot T, et al. Prognostic factors for survival in adult patients with recurrent glioblastoma: a decision-tree-based model. J NeuroOncol. 2018;136:565-76.

15. Binder L, Pieske B, Olschewski M, Geibel A, Klostermann B, Reiner C, Konstantinides S. N-terminal pro-brain natriuretic peptide or troponin testing followed by echocardiography for risk stratification of acute pulmonary embolism. Circulation. 2005;112:1573-9.

16. Schievano S, Capelli C, Young C, Lurz P, Nordmeyer J, Owens C, Bonhoeffer $P$, Taylor AM. Four-dimensional computed tomography: a method of assessing right ventricular outflow tract and pulmonary artery deformations throughout the cardiac cycle. Eur Radiol. 2011;21:36-45.

17. Barker AJ, Roldan-Alzate A, Entezari P, Shah SJ, Chesler NC, Wieben O, Mark $\mathrm{M}$, Francois CJ. Four-dimensional flow assessment of pulmonary artery flow and wall shear stress in adult pulmonary arterial hypertension: results from two institutions. Magn Reson Med. 2015;73:1904-13.

18. Liu M, Guo X, Zhu L, Zhang H, Hou Q, Guo Y, Yang Y, Jiang T. Computed tomographic pulmonary angiographic findings can predict short-term mortality of saddle pulmonary embolism: a retrospective multicenter study. J Comput Assist Tomogr. 2016;40:327-34.

19. Choi KJ, Cha SI, Shin KM, Lim JK, Yoo SS, Lee J, Lee SY, Kim CH, Park $J Y$, Lee WK. Central emboli rather than saddle emboli predict adverse outcomes in patients with acute pulmonary embolism. Thromb Res. 2014:134:991-6.

20. Langendijk JA, Slotman BJ, van der Waal I, Doornaert P, Berkof J, Leemans $C R$. Risk-group definition by recursive partitioning analysis of patients with squamous cell head and neck carcinoma treated with surgery and postoperative radiotherapy. Cancer. 2005;104:1408-17.

21. Lamborn KR, Chang SM, Prados MD. Prognostic factors for survival of patients with glioblastoma: recursive partitioning analysis. Neuro-Oncology. 2004;6:227-35.

22. Mazouni C, Reitsamer R, Rimareix F, Stranzl H, Uzan C, Garbay JR, Delaloge S, Peintinger $F$. The positive non-sentinel status is not the main decisional factor for chemotherapy assignment in breast cancer with micrometastatic disease in the sentinel lymph node. J Surg Oncol. 2012;106:703-7.

23. DeLong ER, DeLong DM, Clarke-Pearson DL. Comparing the areas under two or more correlated receiver operating characteristic curves: a nonparametric approach. Biometrics. 1988;44:837-45. 
24. Hanley JA, McNeil BJ. The meaning and use of the area under a receiver operating characteristic (ROC) curve. Radiology. 1982;143:29-36.

25. Sugiura T, Tanabe N, Matsuura Y, Shigeta A, Kawata N, Jujo T, Yanagawa N, Sakao S, Kasahara Y, Tatsumi K. Role of 320-slice CT imaging in the diagnostic workup of patients with chronic thromboembolic pulmonary hypertension. Chest. 2013;143:1070-7.

26. Sardi A, Gluskin J, Guttentag A, Kotler MN, Braitman LE, Lippmann M. Saddle pulmonary embolism: is it as bad as it looks? A community hospital experience. Crit Care Med. 2011;39:2413-8

\section{Publisher's Note}

Springer Nature remains neutral with regard to jurisdictional claims in published maps and institutional affiliations.

Ready to submit your research? Choose BMC and benefit from:

- fast, convenient online submission

- thorough peer review by experienced researchers in your field

- rapid publication on acceptance

- support for research data, including large and complex data types

- gold Open Access which fosters wider collaboration and increased citations

- maximum visibility for your research: over $100 \mathrm{M}$ website views per year

At $\mathrm{BMC}$, research is always in progress.

Learn more biomedcentral.com/submissions 